\title{
Um Modelo De Interação Adaptável Para Design Participativo Com Crianças Com Deficiência Intelectual
}

\author{
Rallyson dos S. Ferreira \\ Universidade Federal do Amazonas - UFAM \\ Manaus, Amazonas - Brasil \\ rallysonferreira@ufam.edu.br
}

\author{
Thaís H. Chaves de Castro \\ Universidade Federal do Amazonas - UFAM \\ Manaus, Amazonas - Brasil \\ thais@icomp.edu.br
}

\begin{abstract}
RESUMO
Atualmente encontra-se na literatura pesquisas relevantes sobre Design Participativo (DP). Contudo, poucos demonstram iniciativas envolvendo crianças na tenra idade como parte do processo de Design de Interação (DI) para desenvolvimento de software. Isso torna-se mais difícil quando são crianças com transtornos de neurodesenvolvimento, como autistas, que por não terem habilidades linguísticas suficientes não participam da fase de coleta de dados nem de interações posteriores para validação de protótipos. Assim, essas crianças ficam impossibilitadas de expressar suas preferências de design no desenvolvimento de aplicações criadas exclusivamente para esse mesmo público. Consequentemente, a maioria das aplicações tornam-se de propósitos gerais e inapropriadas para crianças neurotípicas. Através do DP é possível minimizar esses problemas utilizando técnicas para melhorar a compreensão linguística dessas crianças e propor abordagens para desenvolver novas aplicações com qualidade e projetadas para suprir suas necessidades. Portanto, este trabalho objetiva desenvolver um modelo de interação específico para auxílio no DI, através de aplicações lúdicas seguindo os preceitos de DP idealizada para maximizar as possibilidades de comunicação, inicialmente com crianças autistas, ao expressarem, através do contexto de design, suas representações de preferências.
\end{abstract}

\section{ACM Classification Keywords}

J.1.1 [Human-centered computing]: Interaction design (ID) Interaction design process and methods.

\section{PALAVRAS-CHAVE}

Design Participativo, Design Adaptável, Transtorno do Espectro Autismo.

\section{Introdução}

O processo de DP é um dos principais métodos para entender as prioridades de um determinado público e tem realizado importantes contribuições criando soluções de alta qualidade e

Permission to reproduce or distribute, in whole or in part, material extracted from this work, verbatim, adapted or remixed, as well as the creation or production from the content of such work, is granted without fee for non-commercial use, provided that the original work is properly credited. IHC 2019 - WORKSHOP DE TESES E DISSERTAÇÕES, Outubro 21-25, 2019, Vitória, Brasil. In Anais Estendidos do XVIII Simpósio Brasileiro sobre Fatores Humanos em Sistemas Computacionais. Porto Alegre: SBC.

() 2019 by the author(s), in accordance with the terms of the Creative Commons Attribution-NonCommercial 4.0 International Public License (CC BY-NC 4.0). formas alternativas de colocar o principal interessado, o usuário final, dentro do Processo de Design (PD) e desenvolvimento de softwares [20]. Dessa forma, existem na literatura trabalhos que demonstram pesquisas relevantes sobre o processo de DP, mas, poucos demonstram interesse em envolver crianças como parte do PD [14]. O problema torna-se ainda mais difícil quando se trata de crianças na tenra idade com problemas de neurodesenvolvimento, como é o caso de crianças autistas, que não possuem condições psicológicas para responderem os questionamentos na fase de coleta de dados por não terem capacidade cognitiva e habilidades linguísticas suficientes [21].

Dessa forma, essas crianças ficam impossibilitadas de expressar suas preferências de design nas aplicações para o desenvolvimento de ferramentas computacionais criadas exclusivamente para pessoas com características de interação semelhante. E com isso, a maioria das aplicações se tornam de propósitos gerais e inapropriadas para crianças com distúrbios cognitivos, pois não se podem obter informações sobre suas predileções de atividades, sons, cores, formas e design adequado para introduzir nos aplicativos [23]. Assim, apesar das dificuldades de incluir crianças no $\mathrm{PD}$ algumas pesquisas reforçam a necessidade do DP para compreender e analisar os dados obtidos da análise de requisitos, principalmente envolvendo crianças com problemas de neurodesenvolvimento na infância [20].

Por meio do processo de DP é possível minimizar esses problemas utilizando técnicas para melhorar a compreensão linguística de um determinado grupo de usuários (crianças com autismo no caso) e propor abordagens para o desenvolvimento de novas aplicações com qualidade e bem projetadas para suprir as suas necessidades, auxiliando e promovendo sua independência, aprendizado e qualidade de vida [12], [21].

Portanto, esta pesquisa objetiva o desenvolvimento de uma abordagem através de aplicações lúdicas seguindo os preceitos de DP adaptada e contextualizada para maximizar as possibilidades de comunicação, inicialmente de crianças autistas, ao expressarem, através do contexto de design, suas representações de preferências. Pretende-se com os resultados contribuir com os designers para adaptar novas formas de introduzir esses usuários no processo de desenvolvimento de software e assim, identificar suas necessidades, habilidades, e consequentemente projetar e adaptar novas ferramentas computacionais a esse público.

\section{Fundamentação Teórica}

Nesta seção demonstramos os principais conceitos relacionados aos objetivos desta pesquisa, catalogados através de uma revisão 
sistemática da literatura, consistindo em uma visão geral sobre DP, DA e design para crianças autistas.

\subsection{Processo de Design Participativo}

Colocar o usuário dentro do processo de DP é importante para entender as suas reais necessidades, principalmente se tratando de crianças com distúrbios cognitivos, coletando preferências de design e adaptando os modelos por meio dos preceitos de DI [20]. Os modelos de DP mostram o desenvolvimento de atividades participativas combinando, misturando, reinterpretando e adaptando técnicas e ferramentas de um conjunto de métodos com base nas características de um determinado grupo de usuário [8]. Sendo assim, algumas pesquisas buscam auxiliar crianças com deficiência descrevendo estruturas, como o IDEA, que explora parcerias de design inclusivas onde crianças australianas surdas ou com deficiência auditiva são facilitadas por outros indivíduos em processos de design [12].

Utilizando abordagens para avaliação participativa com crianças autistas é possível incluir esses usuários em fases de avaliação dedicadas através da co-definição de metas e métodos, processos, conjuntos de coleta de dados e a co-interpretação dos resultados [21]. Neste, foi desenvolvido uma abordagem denominada PEACE (Avaliação Participativa com Crianças Autistas) e os resultados apresentados são promissores. Da mesma forma, algumas pesquisas propõem envolver crianças autistas para registrar e expressar seus próprios interesses dentro de seus contextos de apoio - o lar e a sala de aula, objetivando criar representações com lugares em que as crianças convivem diariamente [23].

Estas pesquisas estão alinhadas aos conceitos propostos neste trabalho, na qual, insere o usuário final dentro do processo de design, compreendendo suas habilidades e suas necessidades, analisando e interpretando suas características de preferências.

\subsection{Modelos de Design Adaptável}

Apesar da importância e da necessidade de desenvolver softwares adaptáveis poucos projetos apresentam soluções para crianças neurotípicas. No entanto, os trabalhos coletados irão dar suporte substancial a esse projeto de pesquisa pela qualidade e por apresentarem métodos viáveis aos objetivos aqui propostos.

Portanto, é importância entender as necessidades de indivíduos com TEA para permitir o design de interfaces adaptáveis abordando múltiplas fontes de heterogeneidade, como habilidades pessoais, ambientes de trabalho e contexto de uso, dada sua interação com as tecnologias [17]. Uma das principais fontes atualmente é a web, onde a maioria das crianças tem facilidade de acesso por meio de dispositivos móveis. Assim, surge a necessidade de trabalhar com modelos de design adaptáveis para web, permitindo se adequar as suas necessidades específicas [15]. Nessa mesma perspectiva foi desenvolvida uma estrutura baseada na compilação de padrões de acessibilidade web, tecnologias web semânticas. Dessa forma, utilizando padrões de recomendações de design para autistas foi possível adaptar ao contexto de cada usuário adequando o sistema as necessidades individuais [16]. Assim sendo, plataformas adaptativas, como o SPRING: (Smart Platform for Research, Intervention and Neurodevelopmental
Growth), buscam se adequar não somente as características de crianças autistas, mas também, ao contexto de diferentes tipos de crianças com neurodesenvolvimento [9].

Relatos de experiências combinando mais de uma abordagem mostram que é possível tornar os artefatos inteligentes o suficiente para adaptar não somente seus elementos de interface, como também a maneira como eles interagem com crianças neurotípicas. Este método foi chamado de Princípios de Design para Interação Humano-Computador (IHC): Research Through Design (RtD), Participatory Design (PD) e Human Centred Design (HCD) [2].

Algumas abordagens, buscam, através de métodos mais simples, por meio de representações do dia a dia (e.g. através de fotos de objetos e locais conhecidos pelo usuário) projetar aplicativos flexíveis e adaptáveis, orientadas para o usuário e que possam ajudar grupos diversificados a estruturar ações do cotidiano [18].

\subsection{Design para Crianças com Autismo}

Propostas de design inclusivo, voltados ao público autista, principalmente crianças, tem sido proposto levando em conta, principalmente, suas necessidades. Dessa forma, esta pesquisa buscou trabalhos para reconhecer, também, suas habilidades com o propósito de melhorar o processo de comunicação desse público. Assim, alguns trabalhos tratam de aspectos sobre preferências de crianças autistas, nesse caso sobre os sons, buscando entender a atenção e as emoções das mesmas ao ouvir sons diferentes. Os resultados podem ser um entendimento de quais são os sons adequadas que podem informar o desenho de aplicações de saúde generalizadas para essa população [7].

Para examinar o nível de deficiência de crianças autistas percebendo emoções através de expressões faciais e movimentos corporais foi apresentado um estudo através de um jogo baseado em IoT para ajudar os indivíduos do NT a "ler" as emoções das crianças com TEA [22]. Da mesma forma, foi proposto um robô integrado para auxiliar na sessão de terapia para aumentar a capacidade de coletar dados relevantes, como atenção, afeto, direção do olhar e gestos, além das medidas convencionais [5].

Modelos ergonômicos e inclusivos também foram propostos para a criação de um ambiente gráfico para uma aplicação educacional interativa de crianças com TEA, a fim de ajudar a identificá-las na expressão facial e no sentimento que as envolve [10].

Por sua vez, identificando aspectos sobre preferências de crianças autistas em relação aos componentes de interface foi desenvolvido um repositório colaborativo para armazenar recomendações de design de interfaces específicas para crianças autistas disponibilizando essa base de dados a desenvolvedores e profissionais da saúde [11].

Algumas ferramentas tecnológicas, como os ambientes virtuais, também podem auxiliar na inclusão de autistas fazendo representação do mundo real, fornecendo às crianças de Alto Funcionamento, seus professores e cuidadores, uma ferramenta de comunicação que os oriente no ensino e na imersão de seus filhos, ajudando-os a se tornarem autossuficientes em rotinas automatizadas [3]. Devido a isso, utilizando modelos físicos, lugares reais, é possível auxiliar na compreensão e incorporação das regras sociais de interação com pares por crianças autistas [1]. 
Um Modelo de Interação Adaptável para Design Participativo com Crianças com Deficiência Intelectual

Visando maximizar as possibilidades de comunicação foi desenvolvido um menu organizacional automático (AOM) para ajudar crianças autistas a melhorar sua capacidade de se expressar e facilitar a solicitação de ajuda [4]. Seguindo nesta mesma ideia, utilizando métodos de realidade aumentada foi idealizado uma Prancha de Comunicação Alternativa e Aumentativa apresentando vantagens em relação a algumas pranchas existentes podendo facilitar a comunicação e a interação não só de autistas, mas também de outras crianças com limitações na comunicação [21]. Métodos para auxiliar no desenvolvimento da linguagem também se mostra uma técnica promissora, uma vez que podem estimular as habilidades de construção narrativas. Assim, foi proposto o LIA, uma aplicação voltada para o público autista. Os testes de validação com o LIA revelaram que se trata de uma aplicação simples e favorável ao aprimoramento da comunicação de autistas [14].

\section{Metodologia da Pesquisa}

Com o objetivo de alcançar os resultados esperados o processo metodológico será composto por cinco etapas definidas na Figura 1. Vale ressaltar que o esquema metodológico idealizado faz parte da avaliação e ainda não foram totalmente definidos.

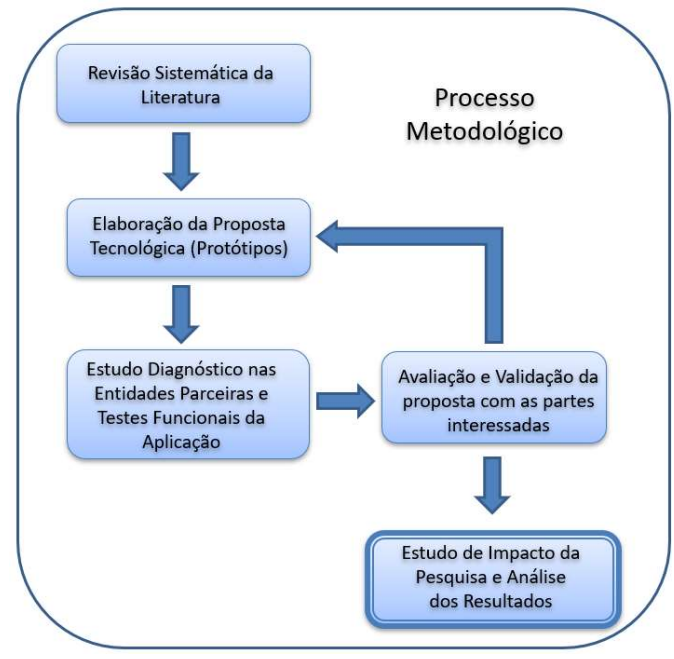

Figura 1: Etapas da Metodologia

\section{Revisão Sistemática da Literatura}

A primeira fase do projeto de pesquisa, uma revisão sistemática da literatura [6], já concluída, foi necessário para realizar uma análise das publicações científicas encontradas atualmente na literatura, objetivando a participação dos usuários dentro do processo de desenvolvimento de software para a caracterização de um modelo de design de interação acessível, visando adaptabilidade individualizada nos artefatos computacionais.

\section{Elaboração da Proposta Tecnológica (Protótipos)}

Através dos resultados da primeira fase, foi idealizado um modelo inicial da proposta tecnológica (protótipo) no qual foi
IHC 2019, Outubro 21-25, 2019, Vitória, Brasil

desenvolvido um aplicativo mobile para que fosse possível realizar os primeiros testes e assim, definir os métodos apropriados para utilizar na avaliação com os usuários finais. Essa fase, já concluída, é importante pois estabelece uma direção a ser seguida na próxima etapa da pesquisa (fase 3 ).

\section{Estudo Diagnóstico nas Entidades Parceiras e Testes Funcionais da Aplicação}

O estudo diagnóstico, fase em andamento, está sendo realizado em parcerias com três entidades que trabalham com crianças autistas, crianças com síndrome de down, entre outros. As entidades estão localizadas na cidade de Manaus no estado do Amazonas e são: Instituto SEMEAR, APAE (Associação de Pais e Amigos dos Excepcionais), e a Escola Municipal Ana Mota Braga. Dessa forma, já foram realizadas duas visitas para aplicação de questionários e entrevistas aos diretores das entidades, aos profissionais que trabalham diretamente com as crianças e aos cuidadores (pais ou responsáveis das crianças). Apesar das dificuldades, devido à falta de disponibilidade dos profissionais e cuidadores para que sejam realizadas as entrevistas, já foram aplicados um número adequado de questionários para realizar os primeiros testes com o protótipo. Ainda nesta fase, será realizado os primeiros testes funcionais da aplicação com crianças autistas.

\section{Avaliação e Validação da Proposta com as Partes Interessadas}

Os resultados alcançados com as três primeiras fases do projeto servirão de base para modelar a proposta tecnológica e apresentar o produto final, baseado principalmente nos modelos catalogados através da revisão sistemática e do estudo diagnóstico nas entidades parceiras. Ainda que não esteja definido, se o produto final será uma aplicação mobile ou um método que será utilizado através de uma ferramenta tecnológica, essa fase ainda será executada, essa etapa será importante para definir: i) os instrumentos de avaliação a serem usados de acordo com os métodos encontrados na literatura e ii) elaboração de um conjunto de características peculiares sobre preferências de crianças autistas no uso de ferramentas computacionais.

Portanto, nesta fase, será realizada a validação da proposta de projeto junto as entidades e os usuários finais. Uma vez que seja aceita a proposta, a pesquisa passa para a sua fase final. Se não for aceita, a proposta volta novamente pela fase de elaboração e modelagem do projeto até que possa ser validada.

\section{Estudo de Impacto da Pesquisa e Análise dos Resultados}

A última fase do projeto de trabalho consiste na avaliação do impacto da implantação da proposta junto as entidades e dos usuários finais (crianças autistas). Esta fase é necessária para constatar se os objetivos e as contribuições, inicialmente propostas, foram alcançadas. Vale ressaltar, que dependendo do tipo de proposta a ser realizada, o estudo poderá ser aplicado de formas diferentes, ainda a ser definido. 


\section{Resultados Parciais}

Os primeiros resultados correspondem a Revisão Sistemática da Literatura, na qual foram consultadas seis bases de dados (IEEE Xplorer, ACM Digital Library, Science Direct, Google Acadêmico, Scopus e Anais do SBIE), utilizando a string em português (("processo de design inclusivo" OR "design inclusivo") AND ("design acessivel" $O R$ "design universal" OR "design para todos") AND ("processo de design adaptável" OR "design adaptativo") AND ("autismo" OR "autista" OR "transtorno do espectro do autismo" OR "transtorno do espectro autista")), e em inglês (("inclusive design process" OR "inclusive design") AND ("accessible design" OR "universal design" OR "design for all") AND ("adaptive design process" OR "adaptive design") AND ("autism" OR "autistic" OR "autism spectrum disorder" OR "autistic spectrum disorder")). No total, foram retornados 3.402 trabalhos, e ao final das etapas de seleção 46 trabalhos no total foram aceitos.

\section{Considerações Finais}

Por meio desta pesquisa espera-se contribuir, principalmente, com o público alvo, inicialmente crianças autistas, pois abrem novas possibilidades de desenvolvimento de artefatos computacionais que irão auxiliá-las em atividades da qual dependem diretamente de outras pessoas para realizarem e agora podem tornar-se mais independente.

Da mesma forma, os resultados possibilitarão aos projetistas de design repensarem as formas de integrarem esses tipos de usuários nos projetos de desenvolvimento de softwares, colocando-os dentro do processo e, assim, ajudando a criar soluções tecnológicas para eles próprios. A pesquisa também contribui para auxiliar na implementação de aplicações mais autônomas e inteligentes, podendo se adaptar às habilidades ou necessidades de cada usuário independentemente, seja ele qual característica possuir ou até mesmo um grupo de usuários.

Portanto, o projeto de trabalho aqui apresentado fortalece as ações de acessibilidade dentro do processo de design participativo, abrindo novas possibilidades de realizar pesquisas dentro do campo de IHC (Interface Humano Computador).

\section{AGRADECIMENTOS}

Nossos agradecimentos as entidades parceiras e a FAPEAM (Fundação de Amparo à Pesquisa do Estado do Amazonas) pelo apoio financeiro dispensados a essa pesquisa.

\section{REFERÊNCIAS}

[1] T. Castro, A. Castro, D. Lima. 2016. A Playground Model to Stimulate Social interaction in Autistic Children. In Proceedings of the 15th Brazilian Symposium on Human Factors in Computing Systems (IHC '16). ACM, New York, NY, USA, Article 57, 4 pages.

[2] T. Castro and D. Lima. 2017. Designing for Children Using the RtD and HCD Approaches". In: Antona M., Stephanidis C. (eds) Universal Access in HumanComputer Interaction. Design and Development Approaches and Methods.

[3] V. Cesário, J. Rodrigues, J. Li, H. Li, I. Wu, and V. Nisi. 2016. Crescendo: Routine Learning App for Children with Autism Spectrum Disorders. In Proceedings of The 15th International Conference on Interaction Design and Children (IDC '16). ACM, New York, NY, USA, 571-576.

[4] C. Chen, C. Wang and C. Su. 2017. The effectiveness of using auto organizational menu to communicate with classmates: a case study of autism spectrum disorders. In Proceedings of the 6th International Conference on Software and Computer Applications (ICSCA '17). ACM, NY, USA, 304-309.

[5] L. A. Dickstein-Fischer, R. H. Pereira, K. Y. Gandomi, A. T. Fathima, and G. S. Fischer. (2017). Interactive Tracking for Robot-Assisted Autism Therapy. In Proceedings of the Companion of the 2017 ACM/IEEE International Conference on Human-Robot Interaction. ACM, New York, NY, USA.

[6] K. R. Felizardo, K. R.; E. Y. Nakagawa, E. Y.; S. C. P. F. Fabri; F. C. Ferrari. 2017. Revisão Sistemática da Literatura em Engenharia de Software: Teoria e Prática; Elsevier: New York City, NY, USA, 2017.

[7] L. Franceli, J. M. Cibrian, E. Lizbeth, and M. Tentori. (2018). A Step towards Identifying the Sound Preferences of Children with Autism. In Proceedings of the 12th EAI International Conference on Pervasive Computing Technologies for Healthcare (PervasiveHealth '18). ACM, New York, NY, USA, 158-167.

[8] C. Frauenberger, J. Makhaeva, and K. Spiel. (2017). Blending Methods: Developing Participatory Design Sessions for Autistic Children. In Proceedings of the 2017 Conference on Interaction Design and Children (IDC '17). ACM, NY, USA, 39-49.

[9] K. Johnson, T. Kristina and R. W. Picard. 2017. SPRING: Customizable, Motivation-Driven Technology for Children with Autism or Neurodevelopmental Differences. In Proceedings of the 2017 Conference on Interaction Design and Children (IDC '17). ACM, New York, NY, USA.

[10] J. Leite, I. Maia, A. Ferreira, L. Rosa. 2019. "Graphic Design of Interactive Tools for People with Autistic Spectrum Disorders". In: Di Bucchianico G. (eds) Advances in Design for Inclusion. AHFE 2018. Advances in Intelligent Systems and Computing, vol 776. Springer, Cham.

[11] A. Melo, J. Santos, L. Rivero, and R. Barreto. (2017). Searching for Preferences of Autistic Children to Support the Design of User Interfaces. In Proceedings of the XVI Brazilian Symposium on Human Factors in Computing Systems (IHC 2017). ACM, New York, NY, USA, Article 45, 10 pages.

[12] T. C. Y. Michelle. 2017. Exploring inclusive design partnerships through an IDEA framework to support deaf or hard of hearing australian children in design process participation. In Proceedings of the 29th Australian Conference on Computer-Human Interaction (OZCHI '17). ACM, New York, NY, USA.

[13] F. M. G. S. C. Moita, L. H. Viana, F. M. Medeiros, V. M. A. Candido. 2017. "Design e desenvolvimento de um game assistivo para autistas". Anais do VI Congresso Brasileiro de Informática na Educação (CBIE 2017) e XXVIII Simpósio Brasileiro de Informática na Educação (SBIE 2017).

[14] E. P. S. Nunes, A. R. Luz, E. M. Lemos. (2016). Approaches of Participatory Design in the Design Process of a Serious Game to Assist in the Learning of Hospitalized Children. HCI, vol. 1, pp. 406-416, 2016.

[15] C. R. Perez, D. A. Mcmeekin., M. Falkmer and T. Tan. 2018. Adaptable maps for people with autism. In Proceedings of the Internet of Accessible Things (W4A '18). ACM, New York, NY, USA, Article 8, 2 pages.

[16] C. R. Perez. 2018. Adaptable user interfaces for people with autism: A transportation example. In Proceedings of the Internet of Accessible Things (W4A '18). ACM, New York, NY, USA, Article 12, 2 pages.

[17] C. R. Perez, D. A. Mcmeekin, M. Falkmer, and T. Tan 2017. Holistic Approach for Sustainable Adaptable User Interfaces for People with Autism Spectrum Disorder. In Proceedings of the 26th International Conference on World Wide Web Companion (WWW '17 Companion). International World Wide Web Conferences Steering Committee, Republic and Canton of Geneva, Switzerland.

[18] K. Ribu and T. Patel. 2016. "Developing a User-Centered Planning Tool for Young Adults with Development Disorders: A Research Based Teaching Project" In H. Petrie et al (Eds) Universal Design 2016: Learning from the Past, Designing for the Future. IOS press 2016.

[19] V. I. Rosa; R. P. Silva; J. L. F. Aymone. 2018. Design inclusivo: processo de desenvolvimento de prancha de comunicação alternativa e aumentativa para crianças com transtorno do espectro do autismo utilizando realidade aumentada. Design e Tecnologia, [S.1.], v. 8, n. 15, p. 51-67, jun. 2018.

[20] D. Satterfield, e M. Fabri. (2017). User Participatory Methods for Inclusive Design and Research in Autism: A Case Study in Teaching UX Design. 186-197. 10.1007/978-3-319-58634-2_15.

[21] K. Spiel, L. Malinverni., J. Good., and C. Frauenberger. (2017). Participatory Evaluation with Autistic Children. In Proceedings of the 2017 CHI Conference on Human Factors in Computing Systems (CHI '17). ACM, New York, NY, USA, 5755-5766.

[22] T. Y. Tang. (2016). Helping Neuro-typical Individuals to "Read" the Emotion of Children with Autism Spectrum Disorder: An Internet-of-Things Approach. In Proceedings of the The 15th International Conference on Interaction Design and Children (IDC '16). ACM, New York, NY, USA, 666-671.

[23] C. Wilson, M. Brereton, B. Ploderer, L. Sitbon, and B. Saggers (2017). Digital Strategies for Supporting Strengths- and Interests-based Learning with Children with Autism. In Proceedings of the 19th International ACM SIGACCESS Conference on Computers and Accessibility (ASSETS '17). ACM, New York, NY, USA, 52-61. 